\title{
Gene Relative Location
}

National Cancer Institute

\section{Source}

National Cancer Institute. Gene Relative Location. NCI Thesaurus. Code C45377.

The location of a feature (for example a SNP, repeat, etc.) relative to the coding sequence of a gene. 